\title{
HER2 Heterogeneity in Gastric Cancer: A Comparative Study, Using Two Commercial Antibodies
}

\author{
Catalin Bogdan Satala, ${ }^{1,2}$ Ioan Jung, ${ }^{1}$ Raluca Ioana Stefan-van Staden, ${ }^{3}$ Zsolt Kovacs, ${ }^{4}$ \\ Calin Molnar, ${ }^{5,6}$ Tivadar Bara Jr., ${ }^{6}$ Zsolt Zoltan Fulop, ${ }^{5,6}$ and Simona Gurzu $\mathbb{D}^{1,2,7}$ \\ ${ }^{1}$ Department of Pathology, George Emil Palade University of Medicine, Pharmacy, Sciences and Technology, \\ Targu-Mures, Romania \\ ${ }^{2}$ Department of Pathology, Clinical County Emergency Hospital, Targu-Mures, Romania \\ ${ }^{3}$ Laboratory of Electrochemistry and PATLAB, National Institute of Research for Electrochemistry and Condensed Matter, \\ Bucharest, Romania \\ ${ }^{4}$ Department of Pathology, George Emil Palade University of Medicine, Pharmacy, Sciences and Technology, \\ Tirgu-Mures, Romania \\ ${ }^{5}$ Department of Surgery, George Emil Palade University of Medicine, Pharmacy, Sciences and Technology, Tirgu-Mures, Romania \\ ${ }^{6}$ Department of Surgery, Clinical County Emergency Hospital, Targu-Mures, Romania \\ ${ }^{7}$ Department of Pathology, Research Center (CCAMF), Tirgu-Mures, Romania
}

Correspondence should be addressed to Simona Gurzu; simonagurzu@yahoo.com

Received 27 August 2020; Revised 8 October 2020; Accepted 12 October 2020; Published 21 October 2020

Academic Editor: Nicola Silvestris

Copyright (C) 2020 Catalin Bogdan Satala et al. This is an open access article distributed under the Creative Commons Attribution License, which permits unrestricted use, distribution, and reproduction in any medium, provided the original work is properly cited.

\begin{abstract}
Background. Although amplification of the gene encoding human epidermal growth factor receptor 2 (HER2) is used as an indicator for response to trastuzumab, the reported response rate is low, and few patients with gastric cancer (GC) benefit from this individualized therapy. The aim of this study was to examine the expression of c-erbB-2 oncoprotein (HER2), in GC samples, using two commercial immunohistochemical (IHC) antibodies, and to validate the results by checking HER2 gene amplification by fluorescence in situ hybridization (FISH). Methods. We assessed the IHC expression of HER2 using the polyclonal antibody from Dako and CB11 clone from Leica, in 93 consecutive cases of GC samples. In all of the cases, FISH analysis was also performed using the BOND-MAX platform. Results. No significant difference was observed between the two HER2 antibodies. Of the 93 cases, $22.58 \%$ demonstrated at least focal and 1+ HER2 positivity. Seven cases (7.53\%) exhibited 3+ expression, and another 7 carcinomas (7.53\%) were equivocal (2+). HER2 amplification was seen in 11 cases $(11.83 \%)$, 10 of which were differentiated adenocarcinomas. In 5 of the cases, $2-5$ sections were examined, which proved the extremely high intratumorally/intraglandular heterogeneity. FISH heterogeneity was higher in cases with only 2+ positivity on IHC assessment, compared with those showing at least one small focus of $3+$ overexpression. HER2 amplification proved to be an independent negative prognostic factor. Conclusions. Due to the highly heterogeneous aspect of GC, at least 3-4 slides should be assessed by IHC, before considering a tumor to be HER2-negative. In cases with small $3+$ foci representing less than $5 \%$ of tumor and in equivocal (2+) cases, FISH analysis remains the gold standard method.
\end{abstract}

\section{Introduction}

Gastric cancer (GC) remains one of the most common causes of cancer-related deaths worldwide, for which the estimation of survival rate, which varies within the same stage, is difficult to be predicted [1]. Although the mortality rate for GC has trended slightly downward in recent decades, it remains a global health problem [2].

Though more than 50 years have passed since the introduction of the Lauren classification, the morphologybased dichotomization of GC into intestinal and diffuse-type carcinoma is still widely used [3]. One of the reasons that an 
effective targeted therapy has not yet been found for GC is its high heterogeneity not only between patients but also within tumors [4-6]. Intratumorally heterogeneity refers to both morphological aspects and immunoreactivity of tumor cells to antibodies detecting specific biomarkers, such as human epidermal growth factor receptor 2 (HER2) [7].

HER2 is a protooncogene located on the long arm of chromosome 17 (17q21), that encodes the transmembrane tyrosine kinase c-erbB-2 oncoprotein, with roles in cellular growth and differentiation. In patients with metastatic GC, HER2 overexpression is used as an indicator of response to anti-HER2 drugs, such as trastuzumab [8-10]. The reasons for the low number of HER2-positive cases and the lack of response to trastuzumab in some positive cases are still unknown. Many commercial antibodies are used for HER2 immunohistochemistry (IHC), and no guideline indicating the number of slides or tumor cells that should be quantified by IHC has yet been implemented.

In this study, we performed an IHC examination of consecutive cases of GC using two anti-HER2 monoclonal antibodies. In cases with inconclusive results, multiple sections were examined, and HER2 gene amplification was assessed.

\section{Materials and Methods}

2.1. Case Selection. Ninety-three consecutive GC cases diagnosed between 2017 and 2020 in the Department of $\mathrm{Pa}$ thology of the Clinical County Emergency Hospital, Targu Mures, Romania, were included in the present study. Criteria of inclusion are as follows: patients who received a curative resection, without preoperative adjuvant therapy, with a diagnosis of gastric adenocarcinoma (G1-G3) and a postoperative survival rate of $\geq 3$ months. Poorly cohesive carcinomas, other histological subtypes of carcinomas, nonepithelial or metastatic tumors, and cases from patients receiving palliative surgery were not included. Processing of the cases was approved by the Ethical Committee of the Clinical County Emergency Hospital, Targu Mures, Romania. Written informed consent for publication of clinicopathological data was obtained from patients, who were prospectively included. The follow-up period was between 8 and 42 months.

For all cases, the available slides with tumor cells were reanalyzed. We aimed to establish the staging according to the most recent edition of the American Joint Committee on Cancer tumor staging manual [11]. Tumors were also staged according to the Dukes-MAC-like staging system, proposed in 2017 [12].

2.2. Immunohistochemistry Analysis and Interpretation. In all cases, conventional slides were used for IHC assessment. After reviewing of the hematoxylin and eosin-stained sections, two experienced pathologists chose one representative sample to be used for further IHC processing. For all 93 cases, we performed immunostaining for HER2 using two monoclonal antibodies from two different manufacturers: Dako (DakoCytomation, Glostrup, Denmark) and
Leica (Leica Biosystems, Germany). We chose the polyclonal antibody c-ErbB-2 (HER2) from Dako and CB11 clone from Leica. High $\mathrm{pH}$ retrieval was performed for the two antibodies. The concentrated antibody from DAKO was diluted $(1: 800)$, but the Leica antibody was ready to use (RTU). Immunostaining was performed automatically (for both antibodies) using the Bond Max fully automated IHC stainer (Leica).

After developing with diaminobenzidine (DAB) and counterstaining with hematoxylin, the membrane expression of HER2 was independently evaluated by two experienced pathologists based on the HercepTest ${ }^{\mathrm{TM}}$ guideline and Ruschoff's criteria [13]: score 0 (negative), tumor cells showed no reactivity or showed reactivity in a site other than the membrane; score 1 (negative), barely visible complete, basolateral, or lateral membranous reaction, visible only at $40 \mathrm{x}$ magnification, in $\geq 10 \%$ of cells; score 2 (equivocal), weak to moderate complete, basolateral, or lateral membranous reaction visible at $10-20 \mathrm{x}$ magnification, in $\geq 10 \%$ of tumor cells; score 3 (positive), strong complete, basolateral, or lateral membranous staining, in $\geq 10 \%$ of tumor cells. In cases in which the results differed between the two pathologists, the case was reevaluated by both pathologists and by the senior pathologist on the team. When necessary, immunostaining was performed on supplementary slides, for elucidation. For cases showing heterogeneous immunostaining (e.g., small areas with $3+$ positivity, below $5-10 \%$, surrounded by areas with $2+$ positivity), the percentage of each grade was determined, and HER2 gene amplification was assessed by fluorescence in situ hybridization (FISH).

2.3. FISH Analysis and Interpretation. To evaluate the grade of HER2 gene amplification and establish an in-house protocol, all HER2-positive tumors, independent of the IHC grade $(1+, 2+$, and $3+)$, were further assessed by FISH. FISH analysis was also performed in samples that showed positivity with only one of the two antibodies.

The FISH technique was automatically performed using the Bond Max fully automated IHC and FISH stainer (Leica). It was performed using the PathVysion HER2 DNA Probe Kit according to the manufacturer's instructions. For interpretation, we used the LSI HER2/neu spectrum orange/ chromosome 17 centromere probe (CEP17)/spectrum green on a Leica CytoVision system based on a Leica DM4000 fluorescence microscope. The analysis considered 30-50 cells from the hotspot, which were chosen at low magnification and then counted at x1000 magnification. Cases with a HER2/CEP17 ratio under 1.8 were considered negative and those with a ratio $\geq 2.2$ were classified as positive. In cases with a HER2/CEP17 ratio between 1.81 and 2.19 and in negative cases, the count was performed again, first by the same pathologist and then by a pathologist experienced in FISH interpretation, in collaboration with a molecular geneticist, with further correlation of results. In addition, in cases that were either negative or equivocal, 50-100 cells were examined for the second interpretation. When necessary, FISH analysis was performed on supplementary slides, for elucidation. 
TABLE 1: Clinicopathological parameters of the included cases.

\begin{tabular}{|c|c|c|}
\hline & $\begin{array}{c}\text { Parameter } \\
\text { Median age (years) }\end{array}$ & $\begin{array}{c}\text { Values }(n=93) \\
67 \pm 11.45(\text { range } 4783)(\%)\end{array}$ \\
\hline Gender & $\begin{array}{c}\text { Males } \\
\text { Females }\end{array}$ & $\begin{array}{l}66(70.96) \\
27(29.04) \\
\end{array}$ \\
\hline Histologic subtype and grade & $\begin{array}{l}\text { Well-differentiated adenocarcinoma (G1) } \\
\text { Moderately differentiated adenocarcinoma (G2) } \\
\text { Poorly differentiated adenocarcinoma (G3) }\end{array}$ & $\begin{array}{c}4(4.30) \\
39(41.93) \\
50(53.76) \\
\end{array}$ \\
\hline Depth of invasion (pT stage) & $\begin{array}{l}\text { pT1 } \\
\text { pT2 } \\
\text { pT3 } \\
\text { pT4 }\end{array}$ & $\begin{aligned} 8 & (8.60) \\
6 & (6.45) \\
24 & (25.80) \\
55 & (59.13)\end{aligned}$ \\
\hline Lymph node status (pN stage) & $\begin{array}{c}\mathrm{pN} 0 \\
\mathrm{pN} 1-3\end{array}$ & $\begin{array}{l}17(18.27) \\
76(81.73) \\
\end{array}$ \\
\hline Distant metastases (pM stage) & $\begin{array}{l}\text { pM0 } \\
\text { pM1 }\end{array}$ & $\begin{array}{l}76(81.73) \\
17(18.27) \\
\end{array}$ \\
\hline Dukes-MAC-like Stage & $\begin{array}{c}A 1 \\
A 2 \\
B 1 \\
B 2 \\
C 1 \\
C 2 \\
D\end{array}$ & $\begin{array}{c}7(7.52) \\
1(1.07) \\
6(6.45) \\
- \\
4(4.30) \\
20(21.50) \\
55(59.13) \\
\end{array}$ \\
\hline
\end{tabular}

TABLE 2: Correlation between immunohistochemical results regarding c-erbB-2 oncoprotein (HER2) expression, using two commercial antibodies and gene status.

\begin{tabular}{ccccc}
\hline & \multirow{2}{*}{ Immunostains } & \multirow{2}{*}{ Total cases (number/\%) } & \multicolumn{2}{c}{ FISH assessment-HER2 gene status (number/\%) } \\
Amplified cases & \multicolumn{2}{c}{ Nonamplified cases } \\
\hline \multirow{2}{*}{ HER2 $3+$} & Dako polyclonal & $7 / 33.33$ & $7 / 100 \%$ & 0 \\
& Leica CB11 clone & $6 / 28.57$ & $6 / 100$ & 0 \\
\hline \multirow{2}{*}{ HER2 2+ $2+$} & Dako polyclonal & $7 / 33.33$ & $4 / 57.14$ & $3 / 42.86$ \\
& Leica CB11 clone & $7 / 33.33$ & $5 / 71.42$ & $2 / 28.58$ \\
\hline \multirow{2}{*}{ HER2 1+ } & Dako polyclonal & $7 / 33.33$ & 0 & $7 / 100$ \\
& Leica CB11 clone & $8 / 38.09$ & 0 & $8 / 100$ \\
\hline
\end{tabular}

2.4. Statistical Analysis. The results were further analyzed using GraphPad Prism 8 (software-free version). The correlation between the overall survival rate, the clinicopathological parameters, and the grade of IHC staining for HER2 was performed using Fisher's exact test and the chi-square test. For all analyses, $p$ values less than 0.05 were considered statistically significant ( $95 \%$ confidence interval).

\section{Results}

3.1. Clinicopathological Parameters. The 93 patients included in the present study were diagnosed with GC between the ages of 47 and 83, and the male-to-female ratio was 2.44. Most of the cases were G2 (moderately differentiated) or G3 (poorly differentiated) adenocarcinomas. Half of the cases ( $n=55 ; 59.13 \%)$ were diagnosed in the advanced stage, pT4N0-3 (Dukes-MAC-like stage D). The other cases were staged as follows: $21.50 \%(n=20)$ as $\mathrm{C} 2(\mathrm{~T} 3 \mathrm{~N} 1-3), 4.30$ $(n=4)$ as $\mathrm{C} 1$ (T3N0), none as $\mathrm{B} 2(\mathrm{~T} 2 \mathrm{~N} 1-3), 6.45 \%(n=6)$ as B1 (T2N0), $1.07 \%(n=1)$ as A2, and $7.52 \%(n=7)$ as A1 (Table 1).
3.2. Immunohistochemical Assessment of HER2. Of the 93 tumors analyzed, $22.58 \%(n=21)$ demonstrated focal positivity of at least $1+$, independent of the antibody used. Only $7.53 \%$ of cases $(n=7)$ was assessed as $3+$ (positive) using the Dako antibody, and $6.45 \%(n=6)$ was assessed as $3+$ using the Leica antibody. In $7.53 \%$ of cases $(n=7)$, the IHC assessment showed $2+$ positivity (equivocal) using both the Dako and the Leica antibodies. The $2+$ category contained the same number of tumors for both clones due to the underscoring tendency of the Leica compared to the Dako antibody: one 3+ case according to Dako assessment was underscored as 2+ using the Leica antibody, and one 2+ case according to Dako was underscored as $1+$ using the Leica antibody. In the category of $1+$ (negative), we identified $7.53 \%$ of cases $(n=7)$ using the Dako and $8.60 \%(n=8)$ using the Leica antibodies (Table 2).

3.3. Fluorescence In Situ Hybridization Assessment of HER2. FISH analysis demonstrated HER2 gene amplification in all cases assessed as 3+ on IHC, while all cases reported as 1+ 

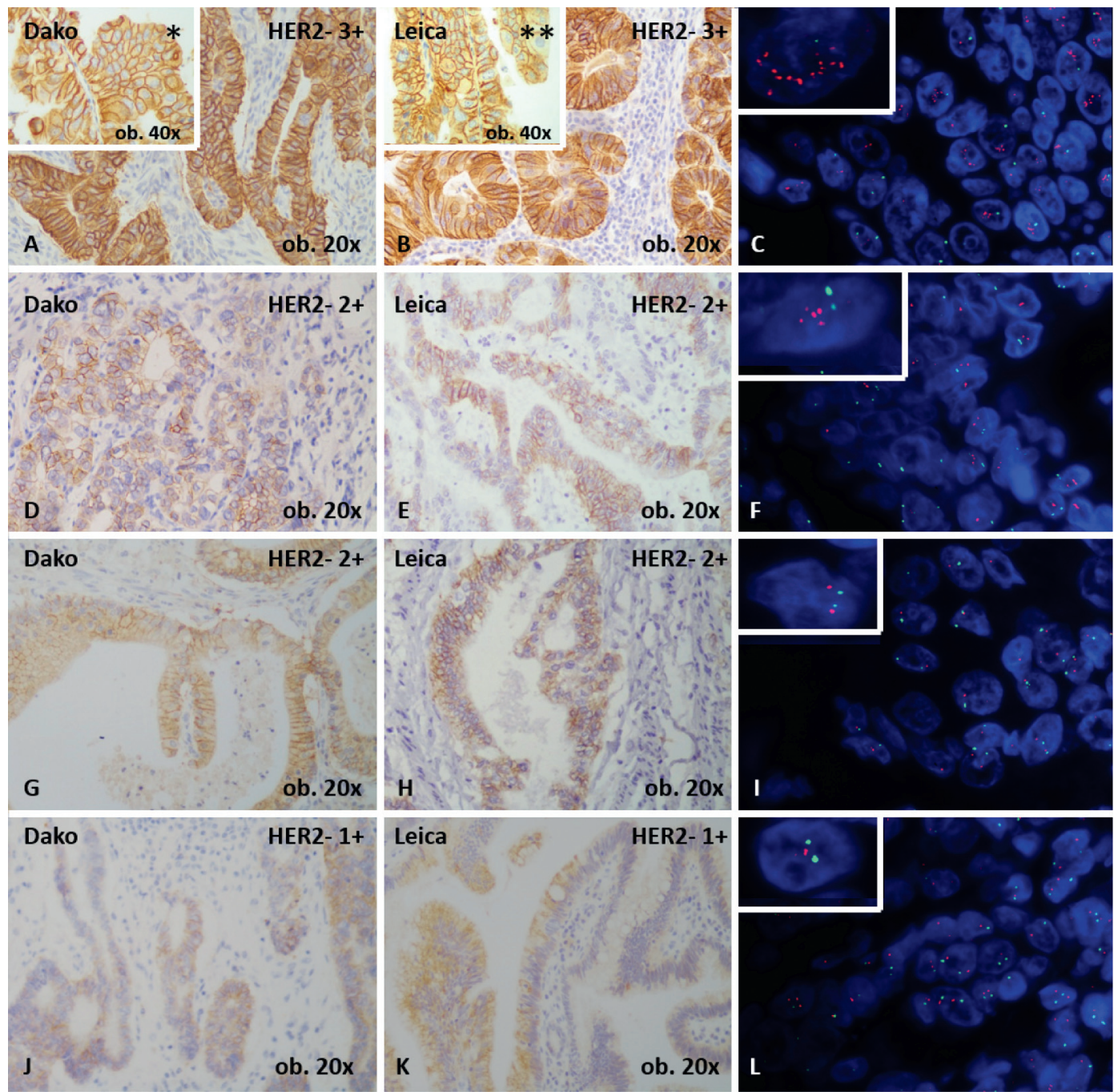

FIgURE 1: Immunoexpression of c-erbB-2 oncoprotein (HER2), revealed with two commercial antibodies and the corresponding FISH examination. In cases with HER2 $3+$ positivity (A and B), with strong, evident, complete membranous staining, and well visible on both 20x and 40x magnification $(*$ and $* *)$, the HER2 gene amplification is clearly proved by FISH analysis (C). In some cases with HER2 2+ positivity (D and E), with moderate complete, membranous reaction visible at 20x magnification, HER2 amplification is present (F), whereas other HER2 2+ cases (G and H) do not show amplification (I), same as the cases assessed as 1+ (J-L).

(negative) on IHC were confirmed to lack HER2 amplification.

The case reported as 3+ on IHC using the Dako antibody and $2+$ using the Leica antibody also showed HER2 gene amplification. Of the 7 cases with equivocal $(2+)$ results using the Dako clone, 4 showed HER2 gene amplification (Figure 1).

While the IHC assessment confirmed HER2 positivity $(3+)$ in $7.53 \%(n=7)$ and $6.45 \%(n=6)$ of cases using the Dako and Leica antibodies, respectively, and $2+$ positivity (equivocal) in another $7.53 \%$ of cases $(n=7)$, HER2 gene amplification was demonstrated in $11.82 \%$ of cases $(n=11)$. The results are summarized in Table 2.

3.4. Intratumorally Heterogeneity. To assess tumor heterogeneity, for five of the cases, we evaluated HER2 expression by IHC and HER2 gene status by FISH on all available slides with viable tumor tissue without extensive necrosis or hemorrhage.

The first two cases were G2 adenocarcinomas with no known distant metastases. Here, 3+ HER2 positivity was found in over $50 \%$ of tumor cells on all four slides examined for each case with both of the antibodies. HER2 gene amplification was confirmed by FISH analysis.

The third case was a G2 adenocarcinoma with hepatic metastases, from which 4 sections from the primary tumor and one from hepatic metastatic tissue were processed. On IHC assessment of this particular case, the first of four tumor sections from the primary tumor demonstrated 3+ HER2 expression on a single focus, representing less than $5 \%$ of the tumor cells, using the antibody from Dako, with the same spot expressing HER2 at a grade of 2+ using Leica assessment. Of the remaining three tumor sections, one demonstrated 2+ HER2 expression using the Dako clone, while the corresponding analysis with the Leica antibody showed 

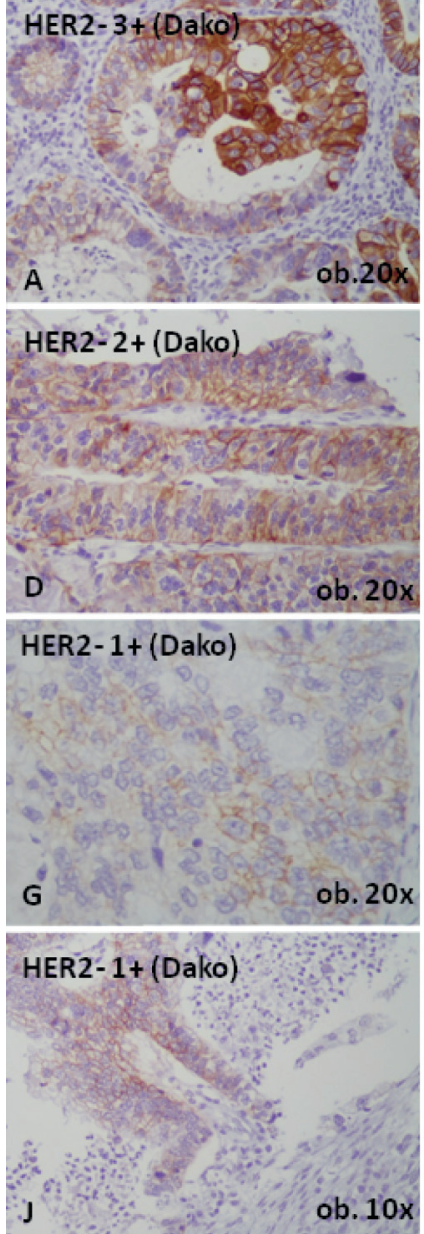
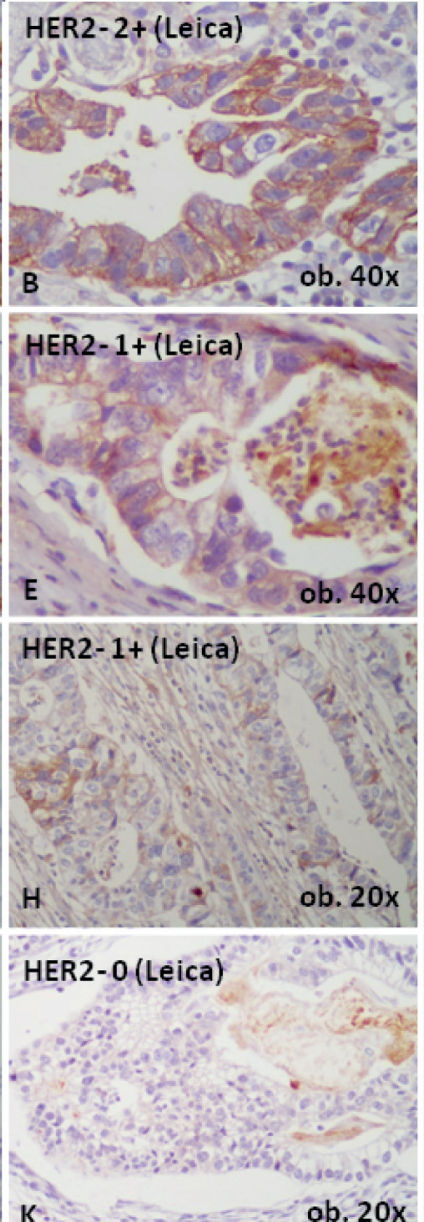
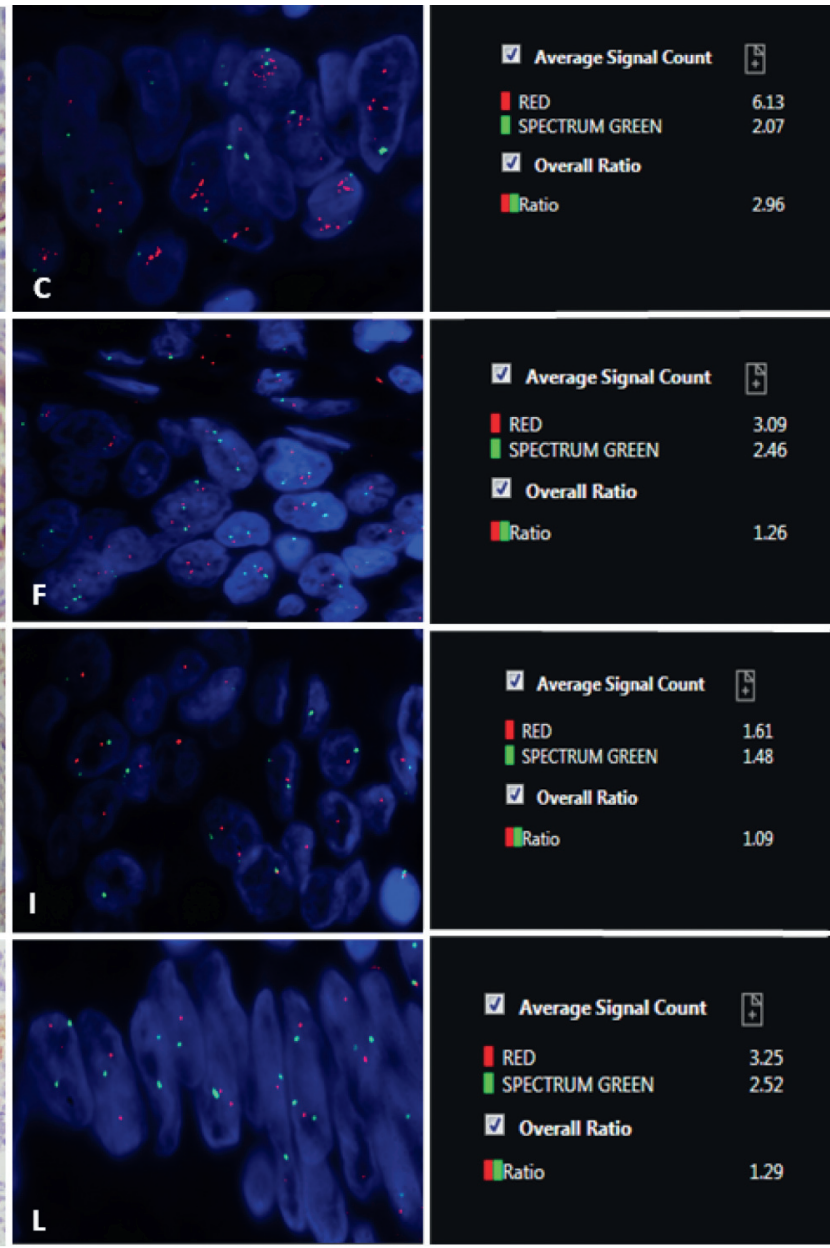

FIGURE 2: Multiple sections from a representative metastatic gastric adenocarcinoma, showing intratumor/intraglandular heterogeneity for HER2 expression and its corresponding FISH expression. One of the slides from primary tumor shows a 3+ focus with Dako (A) which was equivocal (2+) with Leica stain (B) and confirmed as amplified (C) with a HER2/CEP17 ratio of 2.96. In a second slide (D and E), no gene amplification is seen (F), same as for the third section from primary tumor (G-I). The hepatic metastatic tissue also shows no HER2 positivity (J, K) and no gene amplification (L), independently from the used antibody.

only $1+$ expression. The other two sections from the primary tumor were graded as $1+$ using both the Dako and Leica antibodies, and the one section derived from metastatic tumor tissue was negative. FISH analysis demonstrated HER2 amplification only on the first slide that demonstrated positive/equivocal HER2 expression by IHC, with no amplification observed for the remaining slides, including the slide with metastatic tumor tissue (Figure 2).

The fourth case was a G2 adenocarcinoma with no known distant metastases. HER2 assessment by IHC exhibited obviously heterogeneity, with one section showing a focus of $3+$ expression, which represent below $5 \%$ of tumor cells, proved amplified on FISH analysis. The rest of two assessed sections demonstrated equivocal expression on IHC $(2+)$, and they were certified as nonamplified on FISH assessment (Figure 3).

Last, but not least, the fifth case was a G2 adenocarcinoma with multiple regional lymph node metastases $(\mathrm{pN} 3)$, but no known distant metastases. IHC assessment demonstrated a heterogeneous pattern, with one slide with foci of $3+$ expression, below 5\%, which were confirmed as HER2amplified. The second slide showed that multiple areas of $2+$ positivity (over $30 \%$ ) were proved as nonamplified on FISH analysis (Figure 4).

\subsection{Correlation of HER2 Expression with Clinicopathological} Parameters. Examination of the demographic parameters and tumor-related parameters (such as localization, depth of tumor infiltration, lymph node status, lymphovascular invasion, or presence of distant metastases) did not exhibit correlation with the rate of HER2 amplification. Most of the amplified cases (10/11) were differentiated adenocarcinomas (G1/2), with only one of the $50 \mathrm{G} 3$ adenocarcinomas exhibiting amplified HER2 (Table 3). The overall survival rate was also not correlated with the expression of HER2 by 

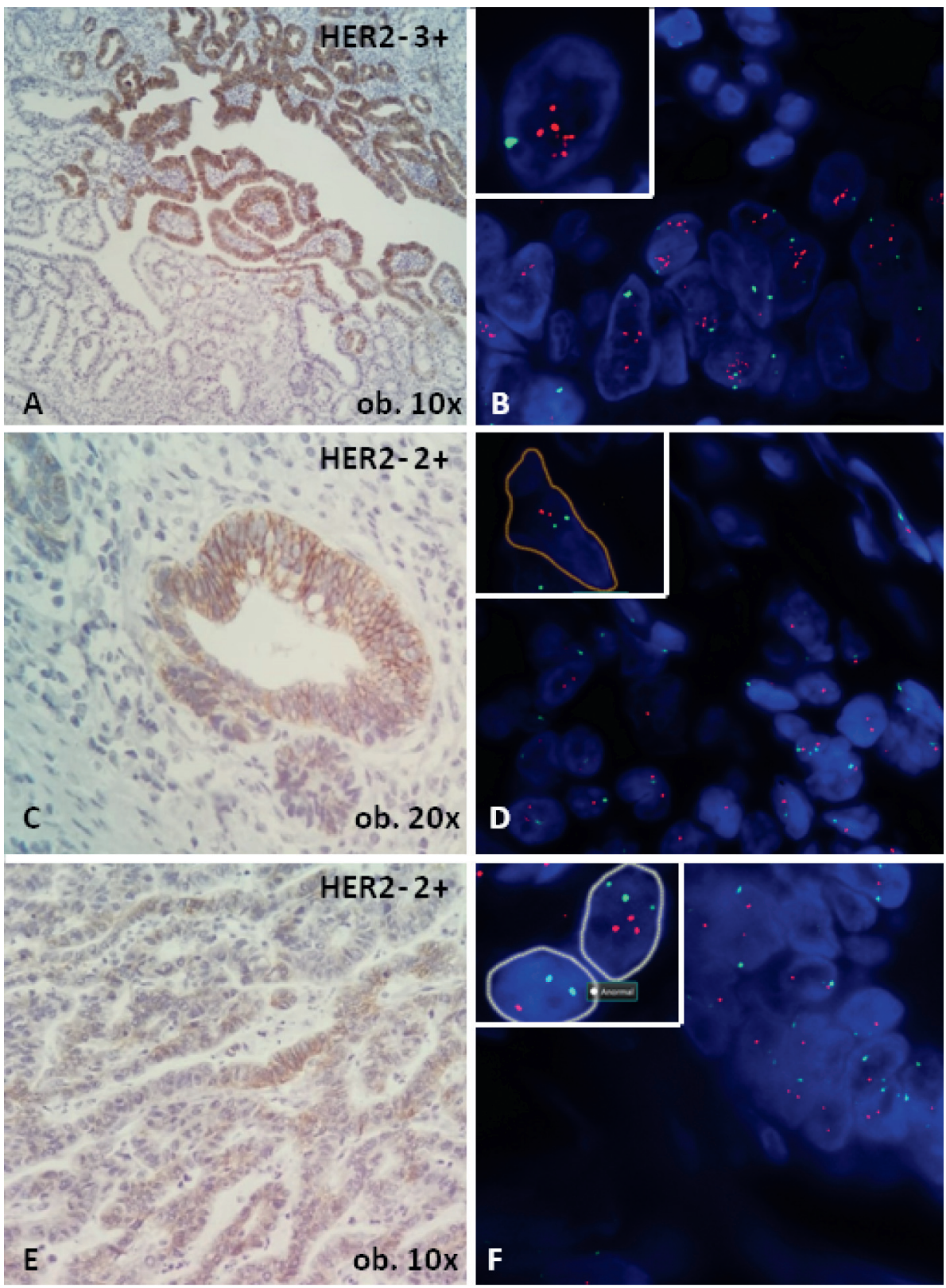

FIGURE 3: Intratumor heterogeneity, revealed by both immunohistochemical and FISH assessment. In one slide, tumor cells exhibit one focus of 3+ expression (A), confirmed as HER2-amplified, with a HER2/CEP17 ratio of 2.41 (B). In other two sections (C-F), extensive areas of $2+$ expression can be seen (C and $\mathrm{E})$, with no HER2 gene amplification (D and $\mathrm{F}$ ).

IHC. In contrast, FISH-verified amplification of the HER2 gene was an independent indicator of worse survival (Figure 5).

\section{Discussion}

Despite improvements in the diagnosis and treatment of patients with GC, the 5-year survival rate is still poor, only $30 \%-35 \%[9,10]$. With many GCs diagnosed every year, the need for standardized prognostic and predictive markers is emphasized in many studies published on this subject; nonetheless, much remains unknown $[14,15]$. Amongst the markers studied in GC, HER2 seems to have the greatest importance not only as a prognostic marker but also because it has therapeutic importance due to the development and use of anti-HER2 therapy $[16,17]$. Trastuzumab is the only anti-HER2 target therapy approved in GC [17], but the selection of patients that could benefit from this treatment is not as straightforward as it is in breast cancer.

The main reason of the difficulty in assessing HER2 in GCs is the intratumorally heterogeneity of its expression, which occurs in $69 \%-75 \%$ of cases $[4-7,9,18,19]$. In this paper, we emphasize and confirm this heterogeneity, which is present in the same tumor, between primary tumor and metastatic tissue and even in the same tumor gland. It is difficult to manage this aspect, as usually only one tumor 


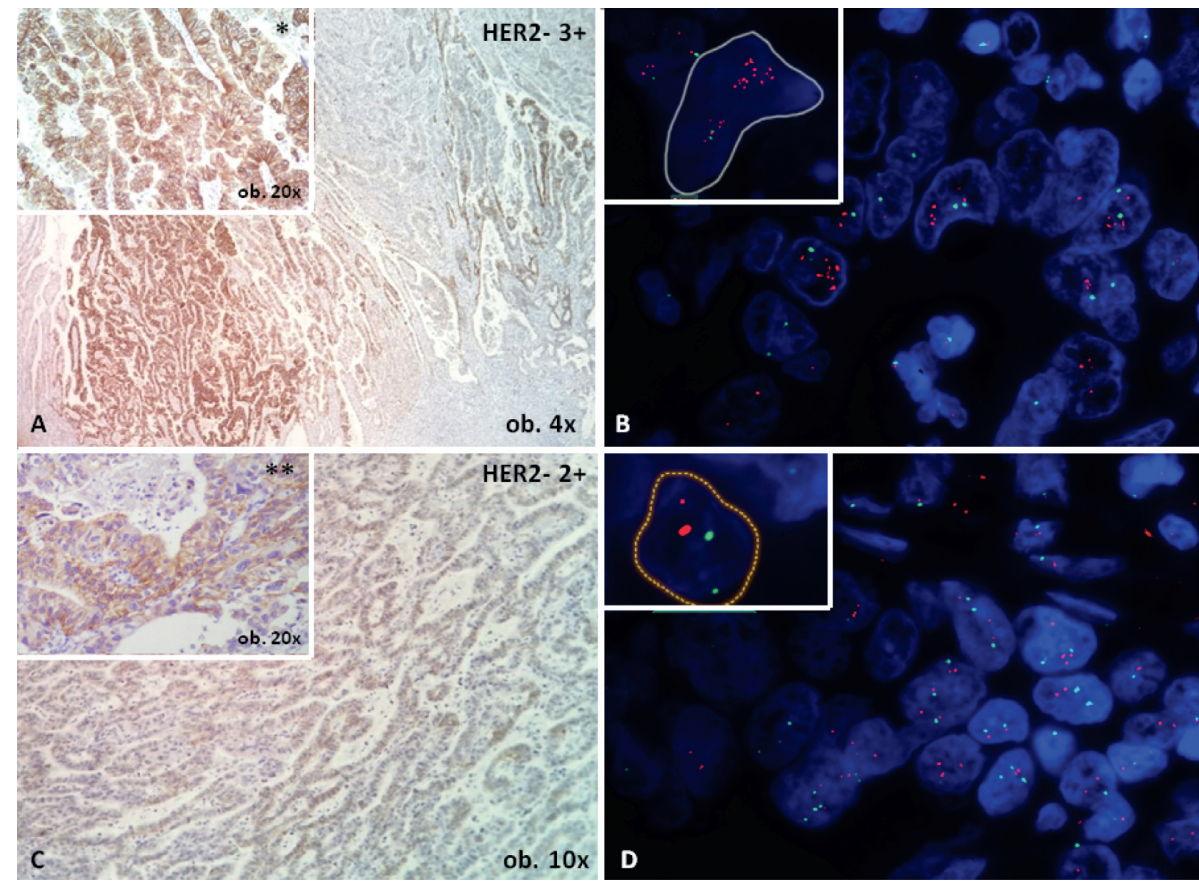

FIgURE 4: Two sections from a gastric adenocarcinoma, with HER2 heterogeneity. On the sample with a single focus of 3+ expression on immunohistochemistry (a), the HER2 gene is amplified, with a HER2/CEP17 ratio of 2.21 (b). On the second sample, with larger areas of 2+ positivity (c), no gene amplification is proved on FISH (d).

TABLE 3: Correlation between clinicopathological factors and HER2 gene status.

\begin{tabular}{|c|c|c|c|c|}
\hline & \multirow{2}{*}{ Parameter } & \multicolumn{2}{|c|}{ HER2 gene status } & \multirow{2}{*}{$p$ value } \\
\hline & & Number of amplified cases & Number of nonamplified cases & \\
\hline Median age (years) & & $73 \pm 11.13$ & $67 \pm 15.32$ & 0.57 \\
\hline \multirow{2}{*}{ Gender } & Male & 7 & 59 & \multirow{2}{*}{0.72} \\
\hline & Female & 4 & 23 & \\
\hline \multirow{2}{*}{ Localization } & Proximal stomach & 5 & 38 & \multirow{2}{*}{1.00} \\
\hline & Distal stomach & 6 & 44 & \\
\hline \multirow{3}{*}{ Histological grade } & G1 & 3 & 1 & \multirow{3}{*}{$<0.001$} \\
\hline & G2 & 7 & 32 & \\
\hline & G3 & 1 & 49 & \\
\hline \multirow{3}{*}{ pT stage } & pT1-2 & 1 & 13 & \multirow{3}{*}{0.64} \\
\hline & pT3 & 4 & 20 & \\
\hline & pT4 & 6 & 49 & \\
\hline \multirow{2}{*}{ pN stage } & pN0 & 3 & 18 & \multirow{2}{*}{0.71} \\
\hline & $\mathrm{pN} 1-3$ & 8 & 64 & \\
\hline \multirow{2}{*}{ pM stage } & pM0 & 9 & 67 & \multirow{2}{*}{1.00} \\
\hline & pM1 & 2 & 15 & \\
\hline \multirow{3}{*}{ Dukes-MAC-like stage } & $\mathrm{A} 1(\mathrm{~T} 1 \mathrm{~N} 0)+\mathrm{B} 1(\mathrm{~T} 2 \mathrm{~N} 0)+\mathrm{C} 1(\mathrm{~T} 3 \mathrm{~N} 0)$ & 3 & 14 & \multirow{3}{*}{0.58} \\
\hline & $\mathrm{A} 2(\mathrm{~T} 1 \mathrm{~N} 1-3)+\mathrm{C} 2(\mathrm{~T} 3 \mathrm{~N} 1-3)$ & 3 & 18 & \\
\hline & $\mathrm{D}(\mathrm{T} 4 \mathrm{~N} 0-3)$ & 5 & 50 & \\
\hline \multirow{2}{*}{ Lymphovascular invasion } & Present & 9 & 51 & \multirow{2}{*}{0.32} \\
\hline & Absent & 2 & 31 & \\
\hline
\end{tabular}

section is used for diagnosis, and the cutoff is $10 \%$. This paper highlights the importance of testing HER2 expression in at least 3-4 slides, especially for differentiated carcinomas that do not show $3+$ positivity on the first slide. Moreover, if tumor cells express $3+$ or $2+$ HER 2 at any extent, even under $5 \%$, on the first slide, it is worth analyzing additional tumor slides for larger foci of HER2 positivity. No cases should be considered HER2-negative without IHC examination of at least 3-4 slides. Biopsy specimens should not be interpreted as negative in any cases, and at least 5 different fragments should be analyzed $[9,20]$.

In this study, $15 \%$ of cases were HER2-positive $(2+$ and $3+)$, in line with literature data that showed relatively wide ranges of HER 2 protein expression (between 5\% and 42\%) [21]. The amplification rate was $11.83 \%$ in this cohort, the reported rate being from $4 \%$ to $13 \%$ [21]. These relatively 


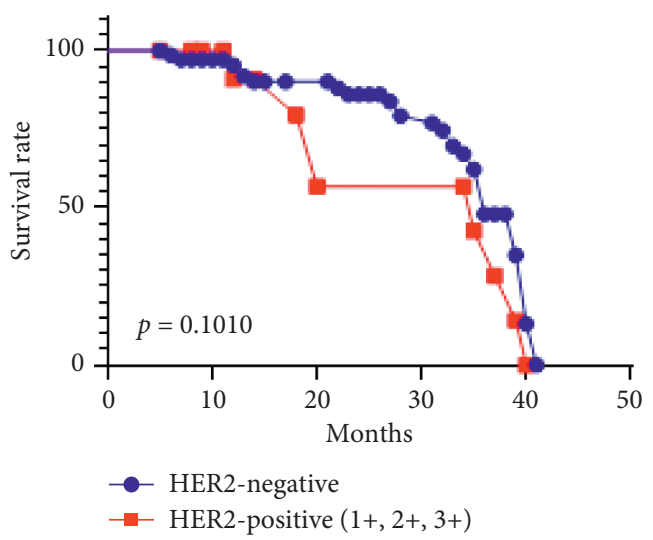

(a)

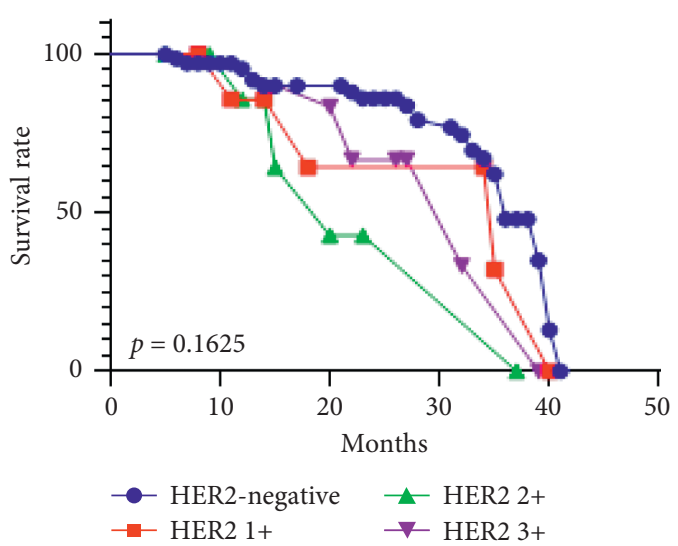

(b)

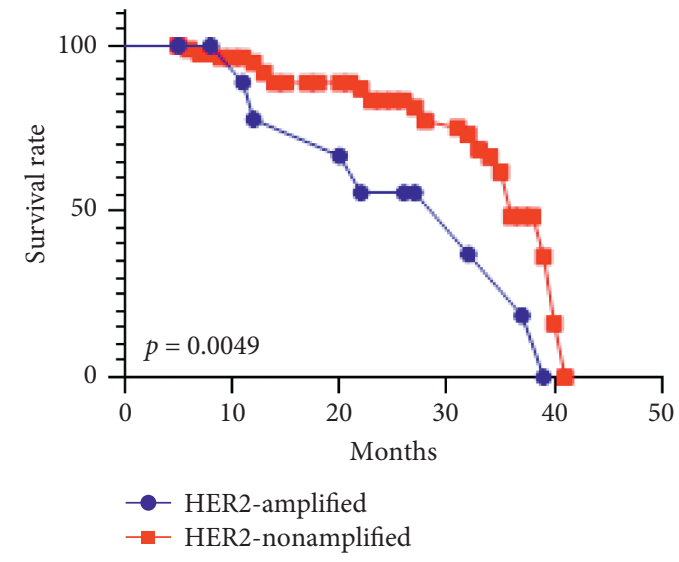

(c)

FIGURE 5: Kaplan-Meier survival curves demonstrate no independent prognostic role of the HER2 immunoexpression (a) and (b), but cases confirmed by FISH with HER2 gene amplification have a considerably lower survival rate, compared to the nonamplified cases (c).

wide ranges are due to the different protocols using, beginning with discrepancies in fixation, use of different antibodies and, maybe most important, use of nonstandardized scoring protocols, especially on FISH analysis. Like our data, it was previously emphasized that the percentage of HER2 overexpression is consistently higher in tumors with well- or moderately differentiated morphology compared to poorly differentiated carcinomas [22-26]. As poorly cohesive carcinomas rarely express HER2, we did not include such cases in this study. However, their inclusion might significantly decrease the reported rate of HER2 positivity.

With the well-known possibility of false-positive/falsenegative results on IHC assessment, we simultaneously evaluated the cases under the study using two different commercial HER2 antibodies. The positivity rate was similar, with only one of the $3+$ cases using the Dako antibody showing equivocal positivity $(2+)$ with the Leica antibody. However, this case showed HER2 amplification. Moreover, all $1+$ cases identified using the Dako antibody were assessed as negative using the Leica antibody. As the correlation between the results obtained using the antibodies from the two manufacturers is over 90\% [21, 26-29], both clones can be safely used in daily diagnosis, but an in-house standardization is mandatory.
An interesting aspect arose regarding the impact of IHC heterogeneity on FISH analysis, which should be performed by an experienced pathologist. In cases that showed at least one focus of $3+$ overexpression on IHC with either of the clones used, with larger areas of 2+ IHC positivity nearby, FISH analysis demonstrated a relatively homogeneous number of amplified HER 2 copies in both the $3+$ and the $2+$ areas. In comparison, in FISH-confirmed positive cases with only $2+$ positivity on IHC assessment, FISH analysis demonstrated intercellular heterogeneity in the number of amplified HER 2 copies. From our perspective, this could have two possible explanations: either the IHC assessment was performed with too much vigilance, with $3+$ areas being misinterpreted as $2+$ areas due to technical difficulties, or the subcellular mechanisms responsible for HER2 amplification are slightly different in cases with $3+$ IHC results compared to those with only $2+$ expression. These aspects should raise the possibility of other molecular signaling pathways acting as positive modulators of classic HER2 gene expression. It was even suggested that a better response to trastuzumab could be obtained in patients whose tumors exhibited 3+ HER2 (quantified using IHC methods and confirmed with HER2 amplification) compared with equivocal (2+) amplified cases [30]. 
One molecular mechanism involved in GC cell heterogeneity could be polysomy, whole chromosomal multiplication [31-34], but this subcellular alteration cannot be responsible for molecular modification in all cases, as FISH analysis does not always report equal amplification of the centromeric region of chromosome 17 (CEP17) and the HER2 gene.

\section{Conclusions}

In GC, the HER2 gene is more frequently amplified in differentiated adenocarcinomas, but the rate of intratumorally heterogeneity is extremely high. To prove HER2 positivity, at least 3-4 slides should be examined, and FISH analysis should be performed in any case that shows clusters of HER2 3+ positivity, even they represent less than $5 \%$ of tumor cells. A gastrointestinal pathologist with experience in FISH analysis should perform interpretation of immunostaining. Both Dako and Leica clones can be successfully used in daily practice. When possible, for FISH analysis, the samples with at least one small focus of $3+$ should be chosen, rather than those with extensive $2+$ positivity. HER 2 amplification is an independent negative prognostic indicator in GC.

\section{Data Availability}

The clinicopathological data used to support the findings of this study are available from the corresponding author upon request.

\section{Conflicts of Interest}

The authors declare that they have no conflicts of interest.

\section{Authors' Contributions}

SCB drafted the paper, performed the FISH examination, contributed to the interpretation of immunohistochemical data, and established the study design; JI supervised the immunohistochemical interpretation and contributed to the study design; SVSRI provided the financial support of the study and participated at samples collections; KZ participated at FISH assessment; MC and BTJ participated at surgical interventions and collecting of clinical data; FZZ participated at collection of clinical data and tissue samples and performed the clinical follow-up; GS supervised the experiment, interpretation of the immunohistochemical and FISH correlations, and gave the final consent for publication.

\section{Acknowledgments}

This work was supported by a grant of the Romanian National Authority for Scientific Research, CNCS-UEFISCDI (project no. 20 PCCF/2018; code. PN-III-P4-ID-PCCF2016-0006). The authors would like to thank the Cambridge Proofreading LLC team who did English proofread. The authors also thank Ms. Genoveva Rigmanyi for the technical support.

\section{References}

[1] S. Gurzu, I. Jung, H. Sugimura et al., "Maspin subcellular expression in wild-type and mutant TP53 gastric cancers," World Journal of Gastrointestinal Oncology, vol. 12, no. 7, pp. 741-755, 2020.

[2] M. R. Silva, A. Alarcao, T. Ferreira et al., "Evaluation of HER2 by automated FISH and IHC in gastric carcinoma biopsies," The International Journal of Biological Markers, vol. 31, pp. e38-e43, 2016.

[3] P. Laurén, "The two histological main types of gastric carcinoma: diffuse and So-called intestinal-type carcinoma," Acta Pathologica Microbiologica Scandinavica, vol. 64, no. 1, pp. 31-49, 1965.

[4] S. W. T. Ho and P. Tan, "Dissection of gastric cancer heterogeneity for precision oncology," Cancer Science, vol. 110, no. 11, pp. 3405-3414, 2019.

[5] B. Lim, J. H. Kim, M. Kim, and S. Y. Kim, "Genomic and epigenomic heterogeneity in molecular subtypes of gastric cancer," World Journal of Gastroenterology, vol. 22, no. 3, pp. 1190-1201, 2016.

[6] T. Chen, X. Y. Xu, and P. H. Zhou, "Emerging molecular classifications and therapeutic implications for gastric cancer," China Journal Cancer, vol. 27, pp. 35-49, 2016.

[7] D.-Y. Oh and Y.-J. Bang, "HER2-targeted therapies - a role beyond breast cancer," Nature Reviews Clinical Oncology, vol. 17, no. 1, pp. 33-48, 2020.

[8] J. N. Gerson, S. Skariah, C. S. Denlinger, and I. Astsaturov, "Perspectives of HER2-targeting in gastric and esophageal cancer," Expert Opinion on Investigational Drugs, vol. 26, no. 5, pp. 531-540, 2017.

[9] T. Kijima, T. Arigami, Y. Uenosono et al., "Comparison of HER2 status before and after trastuzumab-based chemotherapy in patients with advanced gastric cancer," Anticancer Research, vol. 40, no. 1, pp. 75-80, 2020.

[10] Y. Ishimine, A. Goto, Y. Watanabe et al., "Loss of her2 positivity after trastuzumab in her2-positive gastric cancer: is change in her2 status significantly frequent?" Case Reports in Gastrointestinal Medicine, vol. ;2015, p. 132030, 2015.

[11] M. B. Amin, S. Edge, F. Greene et al., AJCC Cancer Staging Manual, Springer, Berlin, Germany, 8th edition, 2017.

[12] S. Gurzu, H. Sugimura, J. Orlowska et al., "Proposal of a Dukes-MAC-like staging system for gastric cancer," Journal of Investigative Medicine, vol. 65, no. 2, pp. 316-322, 2017.

[13] J. Rüschoff, M. Dietel, G. Baretton et al., "HER2 diagnostics in gastric cancer-guideline validation and development of standardized immunohistochemical testing," Virchows Archiv, vol. 457, no. 3, pp. 299-307, 2010.

[14] L. Sisic, D. Vallböhmer, N. H. Stoecklein et al., "Serum microRNA profiles as prognostic or predictive markers in the multimodality treatment of patients with gastric cancer," Oncology Letters, vol. 10, no. 2, pp. 869-874, 2015.

[15] K. Hur, T.-S. Han, E.-J. Jung et al., "Up-regulated expression of sulfatases (SULF1 and SULF2) as prognostic and metastasis predictive markers in human gastric cancer," The Journal of Pathology, vol. 228, no. 1, pp. 88-98, 2012.

[16] W.-H. Kim, L. Gomez-Izquierdo, F. Vilardell et al., "HER2 status in gastric and Gastroesophageal Junction cancer," Applied Immunohistochemistry \& Molecular Morphology, vol. 26, no. 4, pp. 239-245, 2018.

[17] F. De Vita, F. Giuliani, N. Silvestris et al., "Current status of targeted therapies in advanced gastric cancer," Expert Opinion on Therapeutic Targets, vol. 16, no. sup2, pp. S29-S34, 2012. 
[18] Y. Kurokawa, N. Matsuura, Y. Kimura et al., "Multicenter large-scale study of prognostic impact of her2 expression in patients with resectable gastric cancer," Gastric Cancer, vol. 18, no. 4, pp. 691-697, 2015.

[19] S. Motoshima, K. Yonemoto, H. Kamei, M. Morita, and R. Yamaguchi, "Prognostic implications of HER2 heterogeneity in gastric cancer," Oncotarget, vol. 9, no. 10, pp. 9262-9272, 2018.

[20] N. Tominaga, T. Gotoda, M. Hara et al., "Five biopsy specimens from the proximal part of the tumor reliably determine her2 protein expression status in gastric cancer," Gastric Cancer, vol. 19, no. 2, pp. 553-560, 2015.

[21] E. E. Pala, U. Bayol, A. Ozguzer, and O. Akman, "HER2 status in gastric cancer: a comparison of two novel in situ hybridization methods (IQ FISH and dual color SISH) and two immunohistochemistry methods (A0485 and HercepTest)," Pathology - Research and Practice, vol. 209, no. 9, pp. 548-554, 2013.

[22] N. Raj, D. Verma, D. Verma, A. Kumar, P. Rai, and R. N. Rao, "HER2 oncogene amplification and immunohistochemical profiling in gastric adenocarcinoma," Discoveries, vol. 6, no. 4, p. e83, 2018.

[23] J. T. Jorgensen, "Role of human epidermal growth factor receptor 2 in gastric cancer: biological and pharmacological aspects," World J Gastroenterol, vol. 20, pp. 4526-4535, 2014.

[24] M. A. Kim, E. J. Jung, H. S. Lee et al., "Evaluation of HER-2 gene status in gastric carcinoma using immunohistochemistry, fluorescence in situ hybridization, and real-time quantitative polymerase chain reaction," Human Pathology, vol. 38, no. 9, pp. 1386-1393, 2007.

[25] X. Z. Chen, W. H. Zhang, W. Q. Yao et al., "Immunohistochemical Her2 expression not associated with clinicopathological characteristics of stage I-III gastric cancer patients," Hepatogastroenterology, vol. 61, pp. 1817-1821, 2015.

[26] L. Stanek, T. Rozkos, J. Laco et al., "Comparison of immunohistochemistry, four in situ hybridization methods and quantitative polymerase chain reaction for the molecular diagnosis of HER2 status in gastric cancer: a study of 55 cases," Molecular Medicine Reports, vol. 10, pp. 2669-2674, 2014.

[27] E. Y. Cho, A. Srivastava, K. Park et al., "Comparison of four immunohistochemical tests and FISH for measuring HER2 expression in gastric carcinomas," Pathology, vol. 44, no. 3, pp. 216-220, 2012.

[28] L. F. Arbahao-Machado, A. A. dos Anjos Jacome, D. R. Wohnrath et al., "HER2 in gastric cancer: comparative analysis oh three different antibodies using whole-tissue sections and tissue microarrays," World Journal Gastroenterology, vol. 19, pp. 6438-6446, 2013.

[29] J. E. Boers, H. Meeuwissen, and N. Methorst, "HER2 status in gastro-oesophageal adenocarcinomas assessed by two rabbit monoclonal antibodies (SP3 and 4B5) and two in situ hybridization methods (FISH and SISH)," Histopathology, vol. 58, no. 3, pp. 383-394, 2011.

[30] J. S. Pyo, J. H. Sohn, and W. H. Kim, "Concordance rate between HER2 immunohistochemistry and in situ hybridization in gastric carcinoma: systematic review and metaanalysis," The International Journal of Biological Markers, vol. 31, pp. e1-e10, 2016.

[31] J. Rüschoff, W. Hanna, M. Bilous et al., "HER2 testing in gastric cancer: a practical approach," Modern Pathology, vol. 25, no. 5, pp. 637-650, 2012.
[32] J. Cho, J. Jeong, J. Sung et al., “A large cohort of consecutive patients confirmed frequent HER2 positivity in gastric carcinomas with advanced stages," Annals of Surgical Oncology, vol. 20, no. S3, pp. 477-484, 2013.

[33] Y. S. Park, H. S. Hwang, H. J. Park et al., "Comprehensive analysis of HER2 expression and gene amplification in gastric cancers using immunohistochemistry and in situ hybridization: which scoring system should we use?" Human Pathology, vol. 43, no. 3, pp. 413-422, 2012.

[34] K. C. Kim, Y. W. Koh, H.-M. Chang et al., "Evaluation of HER2 protein expression in gastric carcinomas: comparative analysis of 1414 cases of whole-tissue sections and 595 cases of tissue microarrays," Annals of Surgical Oncology, vol. 18, no. 10 , pp. 2833-2840, 2011. 